\title{
A new Baksan Large Neutrino Telescope: the project's status
}

Nikita Ushakov, ${ }^{a, *}$ Almaz Fazliakhmetov, ${ }^{a}$ Albert Gangapshev,${ }^{a}$ Vladimir Gavrin, ${ }^{a}$ Tatyana Ibragimova, ${ }^{a}$ Mahti Kochkarov, ${ }^{a}$ Vladimir Kazalov, ${ }^{a}$ Valery Kuzminov, ${ }^{a}$ Bayarto Lubsandorzhiev, ${ }^{a}$ Arslan Lukanov, ${ }^{a}$ Yuri Malyshkin, ${ }^{b}$ Galina Novikova, ${ }^{a}$ Valery Petkov, ${ }^{a}$ Alexander Shikhin, ${ }^{a}$ Andrei Sidorenkov, ${ }^{a}$ Oleg Smirnov, ${ }^{b}$ Evgeniy Veretenkin, ${ }^{a}$ Dmitry Voronin ${ }^{a}$ and Evgeniy Yanovich ${ }^{a}$

a Institute for Nuclear Research RAS, pr. 60-letiya Oktyabrya 7a, Moscow, Russiy

${ }^{b}$ Joint Institute for Nuclear Research, Joliot-Curie st. 6, Dubna, Russiy

E-mail: nikitaushakoff@gmail.com

A large-volume liquid scintillator neutrino detector is proposed to develop at the Baksan Neutrino Observatory of Institute for Nuclear Research of the Russian Academy of Sciences in the North Caucasus. The detector will be located at the depth of 4700 m.w.e. (meter of water equivalent). A target mass of the detector will be $10 \mathrm{kt}$. This multipurpose detector is being developed to study primarily natural neutrino and antineutrino fluxes namely fluxes of solar neutrinos, geoneutrinos and neutrinos from other astrophysical sources. This project, if implemented, would be a successor of the Borexino experiment and other European projects like LENA. The project is aimed to have a record energy resolution, which along with its location at the large depth and relatively far distance from operating nuclear reactors will allow reaching a record sensitivity to the natural neutrino and antineutrino fluxes. We report in the paper the present status of the project and describe some selective results of the project first stage - the detector prototype with liquid scintillator mass of $0.5 \mathrm{t}$. Results of R\&D for the project second stage with 5 tons of liquid scintillator are presented too.

$37^{\text {th }}$ International Cosmic Ray Conference (ICRC 2021)

July 12th - 23rd, 2021

Online - Berlin, Germany

\footnotetext{
*Presenter
} 


\section{Introduction}

The Baksan Large Neutrino Telescope is a liquid scintillation (LS) neutrino detector with a target mass of $10 \mathrm{kt}$, which is proposed to be constructed in the North Caucasus in the underground zone of the Baksan Neutrino Observatory of the Russian Academy of Sciences (BNO INR RAS) at a depth of about 4700 m.w.e. (meter of water equivalent). This multipurpose neutrino detector will be designed to detect neutrino and antineutrino fluxes from the Sun, Earth and astrophysical sources. The main goal of the project is to measure antineutrino fluxes from beta decays of isotopes of natural radioactive families ${ }^{238} \mathrm{U}$ and ${ }^{232} \mathrm{Th}$, as well as ${ }^{40} \mathrm{~K}$ contained in the interior of the earth (geoneutrinos). Reliable registration of these particles will make it possible to establish the contribution of the energy release from the radioactive decay of these isotopes to the total heat flux of the Earth [1]. Another task of this detector will be the registration of CNO-neutrinos. Measurement of the flux of these neutrinos will make it possible to determine with high accuracy the chemical composition of the solar interior. It is especially relevant in the context of modern difficulties in matching observations of the chemical composition of the photosphere with the data of helioseismology. Also, this detector will be aimed at registration of the isotropic flux of antineutrinos accumulated in the Universe as a result of gravitational collapses of the nuclei of massive stars and the formation of neutron stars and black holes; study of the dynamics of a supernova explosion by recording the intensity and spectrum of a neutrino burst in the case of a supernova explosion with a collapsing core at a distance of up to $200 \mathrm{kpc}$ and registration of the total flux of antineutrinos from all available nuclear reactors on Earth. This project, if implemented, would be a successor of the Borexino experiment [2].

The detector project includes four stages: a prototype with a target mass of $0.5 \mathrm{t}$, a 5 -t prototype, a detector with a target mass of $100 \mathrm{t}$ and a full-scale detector for $10 \mathrm{kt}$. The first stage has now been completed [3]. The 0.5 -t detector prototype, which is located in the laboratory of galliumgermanium neutrino telescope (GGNT), includes two zones. The central zone serving as a target for neutrinos is filled with an ultra-pure LS and is surrounded by a second zone filled with ultra-pure water, which serves to protect against external radioactivity. Linear alkylbenzene (LAB) was chosen as the solvent for LS and poured into an acrylic sphere; 2,5-diphenyloxazole, known as PPO, was chosen as the fluor, and its concentration was chosen to be $2 \mathrm{~g} / \mathrm{L}$. Taking into account the inner radius of the acrylic sphere of $48 \mathrm{~cm}$ and the density of the LAB, the LS mass is $400 \mathrm{~kg}$. Scintillator sphere is surrounded by twenty 10-inch PMTs Hamamatsu R7081-100 with a very high quantum efficiency of about $35 \%$ in the wavelength range of $360-430 \mathrm{~nm}$. The PMTs are placed in four levels of five in a checkerboard pattern at the vertices of a pentagon. The distance from the center of the sphere to the PMTs is about $75 \mathrm{~cm}$. The acrylic sphere and the PMTs are mounted on a stainless steel frame, which is located in a water-filled cylindrical polypropylene tank; the thickness of the water is $70 \mathrm{~cm}$. Water is supplied through a sophisticated water purification and storage system in the laboratory, including the set of filters, quartzing, etc.

This article describes the upgrade of registration system, the measurement of the count rate of the 0.5-t detector prototype, the measurement of the muon flux at the location of the detector and the design of the 5-t detector prototype, the second stage of the project. And also the perspectives of the project are described. 


\section{Registration system upgrade}

Initially, the trigger system of the detector worked as follows. The signals from the PMTs, after the dividing panel, were transmitted to the amplifier, and then to the discriminator. The NIM-signals from the discriminator were transmitted to the adder, where, after summation, they were transmitted again to another discriminator; the threshold adjustment on it made it possible to adjust the required number of triggered channels [3]. This scheme is easy to implement, but it has a number of disadvantages. The major upgrade to the registration system is the use of the programmable logic unit (PLU) CAEN V2495 for the trigger system. Figure 1 shows the upgraded registration system.

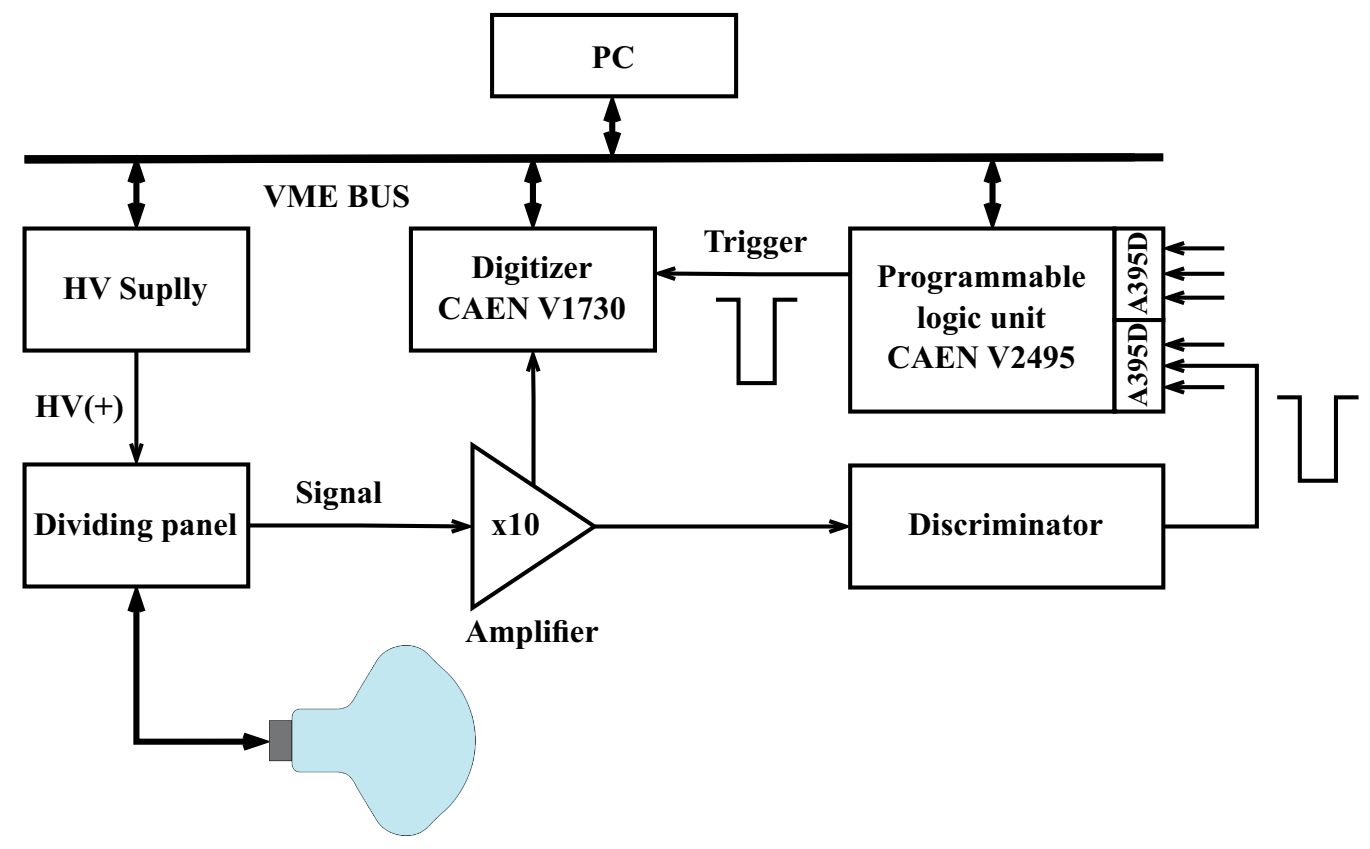

PMTs Hamamatsu R7081-100

Figure 1: Block diagram of the registration system.

This PLU contains two FPGAs programmed in the VHDL language.The first FPGA manages all $\mathrm{I} / \mathrm{O}$, allowing the coincidence/anticoincidence system to be configured using logical operations. This allows you to control which channel was triggered, and combine channels into certain, including overlapping, groups. This allows only time-correlated pairs of events to be taken into account. This is important for a neutrino detector, in which a neutrino event is determined from time-correlated scintillation bursts from a positron and a neutron. Also, the presence of a clock generator in V2495 allows it to be used as a frequency counter to control the counting rates of both the entire detector and each channel (PMT) separately. To control the firmware and to monitor the counting rate of the triggers, software was developed in $\mathrm{C}++$. 


\section{Counting rate measurements}

The measurements were carried out in three stages: with water settled for several months in the water tank, after draining the water and immediately after filling it with water. Measurements for each stage were carried out 1-2 times a day for several days. To stabilize the dark current rate and amplify of the PMTs, measurements were started one day after the high voltage was applied to the PMTs. To register all possible events, the discriminator threshold for all channels was set to 1/4 PE, and the coincidence system was set as follows. The channels were divided into 4 overlapping groups of 10 PMTs: "upper", "lower", "left" and "right". Within the group, the channels were logically multiplied ("AND"), while the groups themselves were logically added ("OR"). Measurements were carried out using the PLU CAEN V2495.

According to the results of measurements, the counting rate of the detector in the established operating mode was $32.5 \mathrm{~Hz}$. In the absence of water protection, the counting rate of detector increased almost 30 times and averaged $927 \mathrm{~Hz}$. Moreover, depending on the day, the counting rate ranged from 915 to $939 \mathrm{~Hz}$. Given the very stable temperature at the location of the detector, these fluctuations can be explained by fluctuations in the concentration of ${ }^{222} \mathrm{Rn}$ in the air; in the presence of water protection, these fluctuations are not observed. Thus, the water protection significantly reduces the number of background events from radioactivity in the space surrounding the detector.

At the third stage, after the tank was filled with water, the counting rate of the detector increased in comparison with that before the water was drained. However, over time, there was a decline in the count rate. Figure 2 shows the dependence of the counting rate of the detector on time, the fit of this dependence and the level of the counting rates before the water is drained. As can be seen from the results obtained, the counting rate of the detector decreased exponentially. The time constant of this decay corresponds to the half-life of ${ }^{222} \mathrm{Rn}, 3.8$ days. Thus, the water purification and storage system needs modernization to purify water from radon.

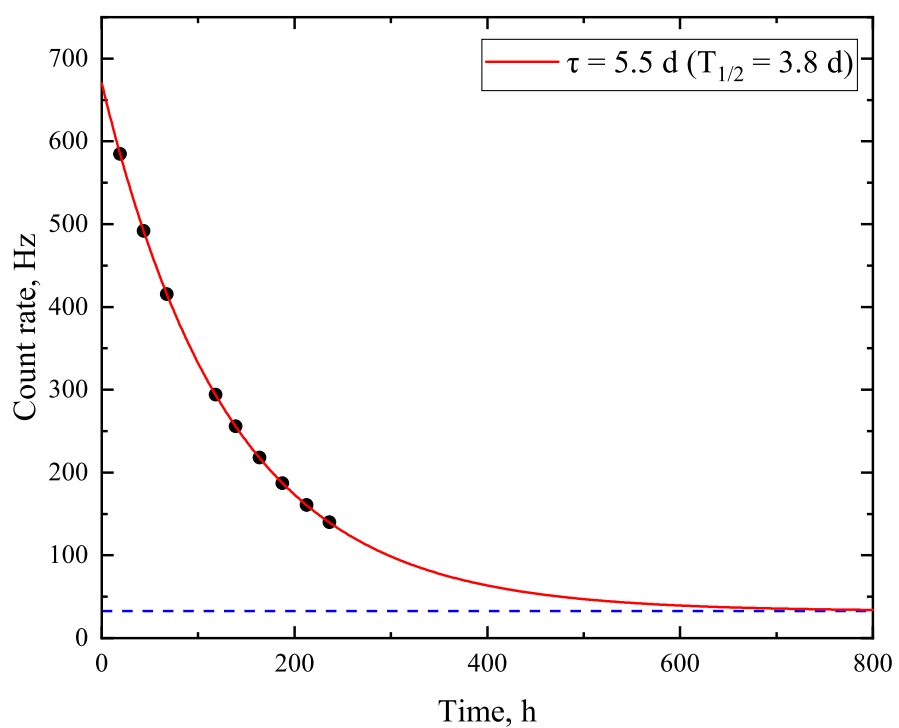

Figure 2: The dependence of the counting rate of the detector on time, the fit of this dependence (red line) and the level of the counting rates before the water is drained (blue line). 


\section{Muon flux measurement}

One of the background sources for neutrino experiments is cosmic ray muons. The ionization loss of a muon when passing through organic scintillators is about $2 \mathrm{MeV} / \mathrm{cm}$. The probability of a muon passing through a point on the plane, perpendicular to its flight path, is evenly distributed over the entire area of the plane. Taking into account the radius of the scintillation target of $48 \mathrm{~cm}$, $99 \%$ of muons passing through it have ionization losses of at least $19 \mathrm{MeV}$. The absolute light yield of the used scintillator is approximately 8000 photons $/ \mathrm{MeV}$. In this case, based on the geometric dimensions of the scintillation target and the quantum efficiency of the PMT, about $37 \%$ at $390 \mathrm{~nm}$, the charge of the signal from the most distant PMT will be over 110 PE.

The threshold of all discriminators was set to the maximum possible level of 6.5 PE to register muon events. The coincidence system was configured in such a way that the trigger was generated only when all 20 PMTs were triggered simultaneously (within the time gate). The measurement time was $797.7 \mathrm{~h}$; a total of 10754 events were recorded. Of these, 88 events were selected, for which the signal charge on any of the channels was more than 110 PE. Figure 3 shows typical pulse waveforms of a muon event and other events. Preliminary results show a flux of muons of $4.28 * 10^{-9} \mathrm{~cm}^{-2} \mathrm{~s}^{-1}$. To clarify the value of the muon flux, it is necessary to increase the measurement time.
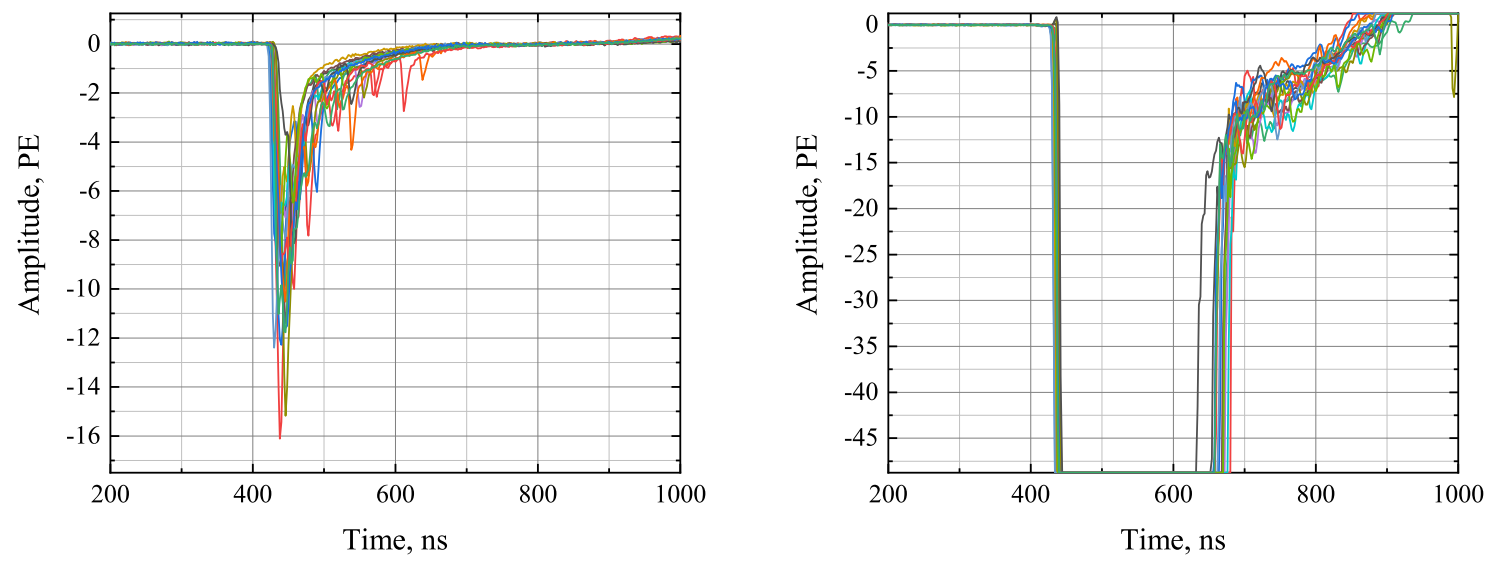

Figure 3: Typical pulse waveforms of muon event (right) and other events (left).

\section{Design of a prototype detector with a target mass of $5 \mathbf{t}$}

The 5-t detector prototype, like the 0.5-t one, is located in the GGNT laboratory and includes two zones. The radius of the acrylic sphere, which is filled with the LS, for the second prototype was increased to $1.1 \mathrm{~m}$. Thus, the volume of the new scintillation target is $5.575 \mathrm{~m}^{3}$, and the mass of the scintillator is about $4.8 \mathrm{t}$. The acrylic sphere fixed on a stainless steel frame is placed in a stainless steel water tank. The diameter and height of the tank are 400 and $400 \mathrm{~cm}$, respectively. The value of water tank is $50 \mathrm{~m}^{3}$, and the thickness of the water has been increased to $90 \mathrm{~cm}$. The acrylic sphere is surrounded by forty-two 10-inch PMTs Hamamatsu R7081-100 located $30 \mathrm{~cm}$ from the sphere to the PMT equators. The main design improvement of the second prototype is the 
use of light concentrators and a muon veto system. Figure 4 shows the 3D-model of the 5-t detector prototype.

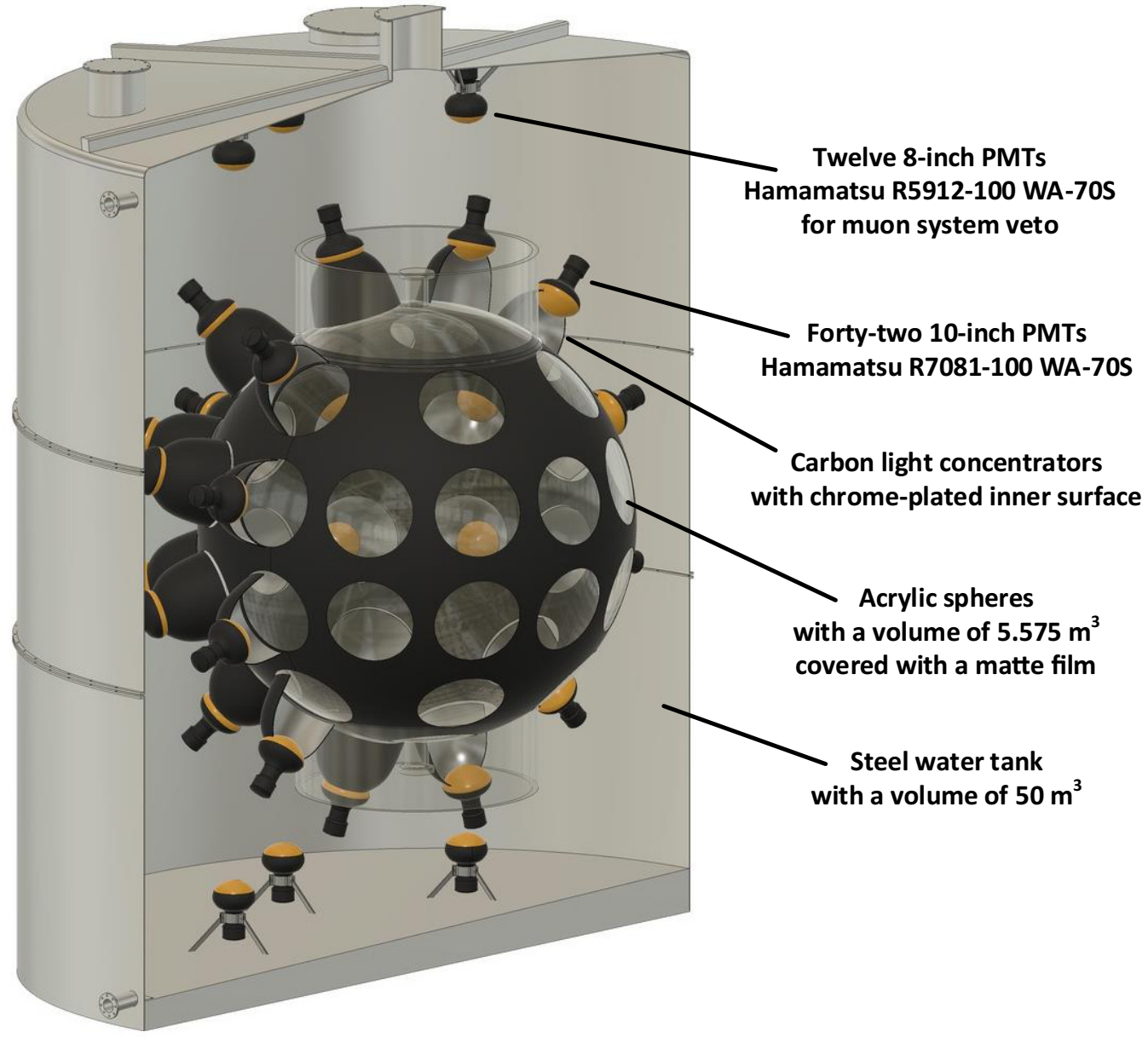

Figure 4: The 3D-model of the 5-t detector prototype.

The concentrators are designed to increase the light collection of the PMTs. The concentrator profile was calculated by the "string method" [4] as it was done for the Borexino [5]. The length of the light concentrator is $30 \mathrm{~cm}$, and the diameter of its base is $40.5 \mathrm{~cm}$. The simulation results show that when the developed concentrator profile is used, the improvement in the light collection of the PMT increases by about 3 times. The figure 5 shows the simulation of the light collection of the concentrator. The concentrators are made of carbon-fiber-reinforced polymer. They are chrome-plated on the inside to reflect light and covered with black matte paint on the outside. Finally, to protect against ultra-pure water, the concentrators are covered with a special varnish.

Muons crossing water emit Cherenkov radiation. Twelve 8-inch PMTs Hamamatsu R5912-100, installed on the bottom and lid of the water tank, are used to register this radiation. According to the results of evaluating these photomultipliers, they have the single photoelectron resolution of $0.67 \pm 0.04$, peak-to-valley ratio of $4.54 \pm 0.52$, transit time spread of $2.08 \pm 0.04$ ns and anode dark count rate of $847 \pm 42 \mathrm{~Hz}$; the quantum efficiency exceeds $30 \%$ in the wavelength range from 


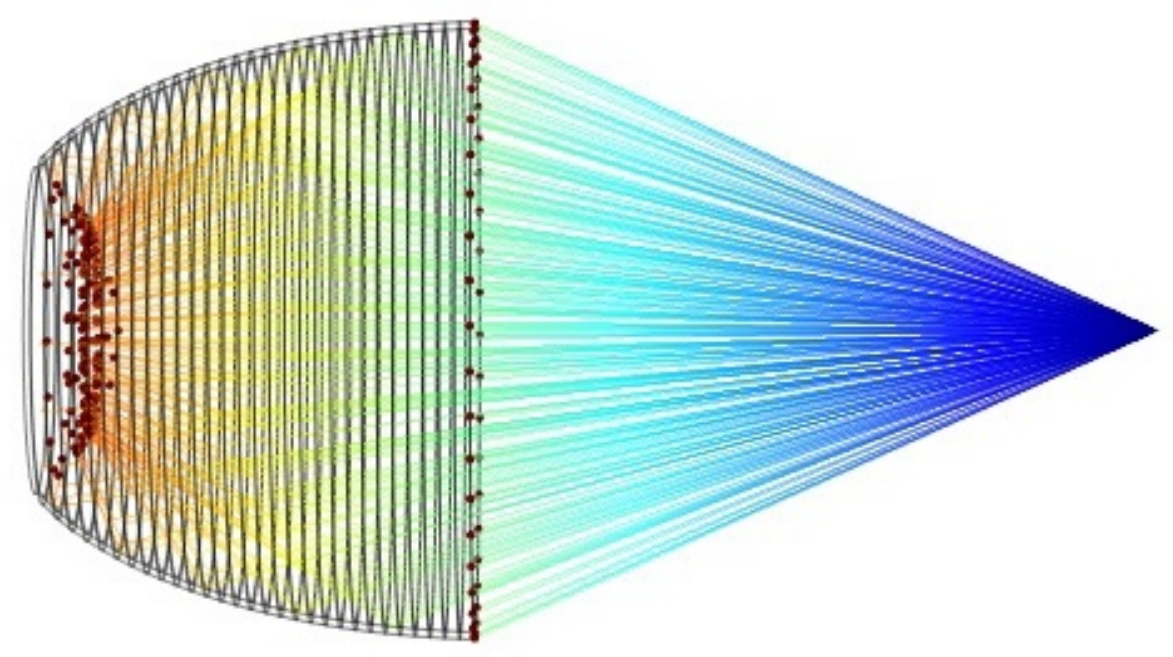

Figure 5: The simulation of the light collection of the concentrator.

$330 \mathrm{~nm}$ to $450 \mathrm{~nm}$ and peaks at almost $40 \%$ at $390 \mathrm{~nm}$. The acrylic sphere is covered with a matte film in the space between the light concentrators for optical separation of the target and water tank volumes. Matt film, like matt paint on concentrators, prevents unwanted light reflections.

\section{Perspectives}

Thus, due to the detection of radon in water that has passed the purification system, this system is currently being modernized. It is supposed to remove radon from water by boiling.

To refine the muon flux and obtain its energy spectrum, in addition to increasing the measurement time, detector data acquisition is performed at a reduced high voltage for the PMTs.

The 5-ton detector prototype is currently under construction. Measurements of the radioactive background of the detector construction elements (frame elements, PMTs, light concentrators, etc) are carried out. A special design has been developed for placing a radioactive source in the center of the acrylic sphere for energy calibration of the $0.5-\mathrm{t}$ and 5-t detectors. The purification of LAB from 9-methylanthracene, $\mathrm{U}$ and $\mathrm{Th}$ by the sorption method using $\mathrm{Al}_{2} \mathrm{O}_{3}$ has begun for the 5-ton detector; PPO is purified from $\mathrm{K}$ by the centrifugation method. In addition, scintillators using new fluors such as POPOP, BPO, Bis-MSB and Butyl PBD are being developed for the 5-t detector and the next stages of the project.

Finally, a special system is being developed to protect the PMTs of the 5-t detector from the effect of a magnetic field. An external magnetic field degrades the timing characteristics of the PMTs, which has a particularly bad effect on the ability to determine the coordinates of an event in the LS target. There are two approaches to ensuring the magnetic protection of PMTs in detectors. The first is to shield each PMT with a material with a high magnetic permeability, for example, permalloy; this approach was used in the Double Chooz [6] and Borexino. Another approach is to surround the entire detector with current conductors like Helmholtz coil. The disadvantage of using permalloy is the change in its magnetic characteristics after even minor deformations; for this reason, 
it is impractical to surround the entire detector with such material. The second method is more difficult to design, but easier to implement. This approach was used in the Super-Kamiokande [7] and is proposed for our project.

\section{Acknowledgments}

The work was supported by the Russian Science Foundation, project No. 17-12-01331.

\section{References}

[1] L. Ludhova and S. Zavatarelli, Studying the Earth with Geoneutrinos, Adv. High Energy Phys. 2013 (2013) 425693.

[2] M. Agostini et al., Spectroscopy of geoneutrinos from 2056 days of Borexino data, Physica B 355 (2005) 116.

[3] N.A. Ushakov et al., New large-volume detector at the Baksan Neutrino Observatory: Detector prototype, J. Phys. Conf. Ser. 1787 (2021) 012037.

[4] L. Oberauer, . Grieb, F. von Feilitzsch and I. Manno, Light concentrators for Borexino and CT, Nucl. Instrum. Methods Phys. Res. A 530 (2004) 453.

[5] R. Winston, J. Minano and P. Benitez, Nonimaging Optics, Academic Press (2005).

[6] E. Calvo et al., Characterization of large-area photomultipliers under low magnetic fields: Design and performance of the magnetic shielding for the Double Chooz neutrino experiment, Nucl. Instrum. Methods Phys. Res. A 621 (2010) 222.

[7] S. Fukuda et al., The Super-Kamiokande detector, Nucl. Instrum. Methods Phys. Res. A 501 (2003) 418. 